\title{
Doença de Crohn e sua relação com a Nefrolitíase: Tratamento Nutricional
}

\section{Crohn's Disease and its Relation with Nephrolithiasis: a Nutritional Treatment}

\author{
Nívea Veiga Almeida ${ }^{1}$ \\ Alden Santos Neves ${ }^{2}$
}

Artigo

Original

Original

Paper

Palavras-chave:
Doença de Crohn

Nefrolitíase

Hiperoxalúria

\section{Resumo}

ADoença deCrohn(DC)éuma doença inflamatória intestinal de causa desconhecida que acomete pessoas de 20 á 50 anos de idade de ambos os sexos e está associada á manifestações extraintestinais secundárias ao processo inflamatório de base, sendo a nefrolitíase a manifestação urogenital mais comum acometendo de 12 a $28 \%$ dos pacientes, devido a alterações na mucosa intestinal e, consequentemente, má absorção, esses cálculos estão associados à hiperoxalúria, além de outras alterações metabólicas associadas. Por esse motivo torna-se importante o conhecimento das alterações metabólicas existentes, para que se possa oferecer o acompanhamento nutricional adequado, e, em específico, no presente estudo, o tratamento nutricional oral que objetiva o controle da ingestão de glutamina, ácidos graxos ômega 3, proteínas, citrato, fibras, magnésio, líquidos, probióticos, fibras solúveis, oxalato e citrato, aumentando assim uma melhor qualidade de vida para estes pacientes. Objetivo:descrever a relação existente entre a DC e Nefrolitíase, e analisar o tratamento nutricional mais adequado.Metodologia: revisão bibliográfica de artigos científicos e sites oficiais. Conclusão: apesar de existir relação entre as doenças citadas, há necessidade de maiores estudos, pois não existem ainda diretrizes nutricionais para nefrolitíase em pacientes DC.

\begin{abstract}
Crohn's disease is an intestinal inflammatory disease of unknown cause that affects people from 20 to 50 years of age on both sex and is associated to extra-intestinal manifestations and to the basic inflammatory process, which nephrolithiasis is the most common urogenital manifestation affecting 12 to $28 \%$ of the patients, due to alterations in the intestinal mucosa and, consequently, bad absorption, these are associated to Hyperoxaluria and other metabolic alterations. For this reason the knowledge of the existing metabolic alterations becomes important, in order to offer an adequate nutritional treatment, and specifically in the present study, the oral nutritional treatment that aims to control the ingestion of glutamine, greasy acid Omega 3, proteins, citrate, fibers, magnesium, liquids, probiotics, staple fibers, oxalate and citrate, thus increasing these patients quality of life. Aim: describe the relationship between DC and nephrolithiasis and analyze the most adequate nutritional treatment. Methodology: review of scientific articles and official sites. Conclusion: Although there is a correlation between the diseases mentioned, there is a need for further studies, because there aren't nutritional guidelines for nephrolithiasis in patients DC.
\end{abstract}

Key words:

Crohn's disease

Nephrolithiasis

Hyperoxaluria
Recebido em 05/2011

Aprovado em 08/2011

\footnotetext{
Graduada em Nutrição (Ciências da Saúde - UniFoa)

${ }^{2}$ Professor Orientador (Ciências da Saúde - UniFoa)
} 
A doença de Crohn (DC) é uma das principais formas de doença inflamatória intestinal, que, segundo estudo realizado em um hospital universitário da região sudeste do Brasil, acomete pessoas de 20 á 50 anos de idade em ambos os sexos ${ }^{1}$.

Essa doença acomete pessoas de diferentes classes socioeconômicas, e nacionalidade, afetando aproximadamente 1,4 milhões de pessoas nos Estados Unidos ${ }^{2}$.

No Brasil, de acordo com Associação Brasileira de Colite Ulcerativa e Doença de Crohn (ABCD), ainda não se sabe a incidência exata de Doença Inflamatória Intestinal (DII).

Uma possível explicação para esse fato seria que, em nosso meio, essas doenças não são consideradas de notificação compulsória, o que nos leva a pensar que as DII podem não estar sendo diagnosticadas, uma vez que os prontuários médicos registram altas taxas de diarreias de etiologia bacteriana ou parasitária ${ }^{3}$.

A etiologia da DC não é completamente conhecida, mas parece envolver interações de fatores comportamentais, microflora do hospedeiro, predisposição genética acometendo resposta imune anormal ou autoimune na parede intestinal. Tem evolução crônica, e gera repercussões importantes na qualidade de vida dos doentes ${ }^{4}$.

Devido a alterações na mucosa intestinal, a formação de cálculo renal é favorecida tornando-a mais comum em relação à população geral, em que a frequência em pacientes com DC varia de 12 a $28 \%$, enquanto que na população em geral afeta 1 a 14\% das pessoas 5 .

A formação de cálculos no trato urinário é um processo multifatorial que envolve o balanço entre os fatores promotores da formação de cálculo e os inibidores da cristalização ${ }^{6}$.

Cálculos urinários podem ser detectados em um número significativo de pacientes com DC, principalmente os que se submetem á cirurgia de ressecção parcial do intestino delgado, sendo esses cálculos associados à hiperoxalúria, hipomagnesúria e hipocitratúria, além de outras alterações metabólicas associadas?

Por esse motivo torna-se importante o acompanhamento nutricional desses pacientes, com adequado conhecimento sobre as alterações metabólicas e a devida correção desses distúrbios, evitando assim, além de possíveis exacerbações da DC, a formação de cálculos renais, condição por si só bastante agressiva e que desencadeia alterações importantes na vida das pessoas por ela acometidas.

Devido ao grande número de complicações encontradas em portadores de Doença de Crohn no Brasil, este estudo se justifica pelo fato de que a relação desta doença com a formação de litíase renal ainda está pouco esclarecida e muitos estudos ainda são necessários para se estabelecer esta relação e melhorar a qualidade de vida dos pacientes acometidos pela doença.

O objetivo do presente estudo é compreender a relação existente entre a Doença de Crohn e Nefrolitíase, sendo objetivos específicos descrever a fisiopatologia dessa relação e relacionar o tratamento nutricional por via oral mais adequado.

\section{Metodologia}

Este trabalho baseou-se em revisão bibliográfica de artigos científicos indexados em bases de dados da internet, como MedLine, LILACS e SCIELO, e sites oficiais, livros e periódicos, compreendendo principalmente o período de 1999 a março de 2011. Foram utilizados como palavras-chave os termos "Doença de Chron", "nefrolitíase","hiperoxalúria" e "tratamento nutricional da Doença de Chron".

\section{Doença de Crohn e Nefrolitíase}

A DC é uma manifestação intestinal inflamatória que acomete igualmente pacientes de 20 a 50 anos de ambos os sexos podendo envolver qualquer parte do trato gastrintestinal (TGI), da boca ao ânus. Sua causa é desconhecida, porém, a predisposição genética e o fenômeno autoimune estão envolvidos. Até o momento, o principal fator de risco para DII é ter história familiar positiva em um parente de primeiro grau. Os gatilhos para o estabelecimento inicial da doença e exacerbações subsequentes são desconhecidos, mas, provavelmente, envolvem interações virais ou bacterianas com células imunes que recobrem a parede mucosa do trato intestinal ${ }^{8}$. 
O intestino delgado e, especialmente, o íleo terminal estão envolvidos em cerca de $75 \%$ dos casos e apenas cerca de 15 a $25 \%$ dos casos envolvem apenas o cólon. Nos segmentos do intestino envolvidos, a inflamação pode ser contínua, não atingindo certas áreas, sendo o envolvimento da mucosa transmuda na medida em que afeta todas as camadas da mucosa intestinal ${ }^{4}$.

A atividade inflamatória crônica recorrente reflete uma resposta apropriada a um estímulo persistentemente anormal ou uma resposta anormalmente prolongada a um estímulo normal. A forte resposta inflamatória em excesso, com envolvimento local e sistêmico dos leucócitos, resulta na liberação de proteases, prostaglandinas, leucotrienos, eicosanoides e radicais de oxigênio livre. Tal resposta parece ser grandemente responsável pelo dano ocorrido no tecido gastrointestinal o que modifica a permeabilidade da parede intestinal às moléculas de alimento e fragmentos de células ${ }^{8}$. Portanto, há evidências de que o aumento da permeabilidade intestinal ocorre antes do estabelecimento da inflamação com marcante significado etiopatogênico ${ }^{9}$.

As manifestações clínicas são decorrentes tanto do acometimento do trato gastrointestinal pela doença, como pelo surgimento de manifestações extraintestinais, que ocorrem em cerca de $35 \%$ dos pacientes ${ }^{10}$.

Ainda no mesmo estudo, ao analisar o perfil dos pacientes com DC, os autores verificaram que a nefrolitíase foi a complicação urogenital mais comum.

Para confirmação, foram observados 40 pacientes com DC estudados; 18\% apresentaram litíase renal e os autores concluíram que esse resultado é mais comum em pacientes que foram submetidos à cirurgia de ressecção parcial do intestino, sendo que, quanto maior for a ressecção, maior a formação e a quantidade de cálculos formados, e que o aparecimento de cálculos ocorre aproximadamente após 2 anos de cirurgia ${ }^{7}$.

A complicação secundária mais frequente em portadores de DC é a nefrolitíase sob a forma de oxalato de cálcio e ácido úrico ${ }^{11}$.

A formação de cálculos no trato urinário é um processo multifatorial que envolve o balanço entre os fatores promotores da formação de cálculo e os inibidores da cristalização, ou seja, quando os mecanismos de inibição estão reduzidos ou ausentes, fatores físicos - químicos que governam a litogênese- transformam a urina de saturada em supersaturada, levando á nucleação, cristalização e ao crescimento do cristal $^{6}$.

A DC e outras doenças inflamatórias intestinais têm sido associadas á nefrolitíase, devido, principalmente, à hiperoxalúria entérica, por consequência da má absorção de gorduras, levando a hiperabsorção de oxalato da dieta $^{12}$.

Em indivíduo normal de 3\% a 5\% do oxalato ingerido são absorvidos, principalmente no cólon, sendo a hiperoxalúria definida pela excreção de oxalato maior que $40 \mathrm{mg} / \mathrm{dia}^{13}$.

Pesquisadores verificaram a excreção de 49,2 $\mathrm{mg} / 24 \mathrm{~h}$ de oxalato em pacientes que apresentaram litíase renal após ressecção parcial do intestino delgado ${ }^{7}$.

A formação de cálculos de oxalato de cálcio é devido à maior absorção e excreção urinária de oxalato, que normalmente não é absorvido quando ligado ao cálcio no intestino, porém, em casos de má absorção ou ressecção cirúrgica do intestino, permanece livre devido á fixação do cálcio aos ácidos graxos, podendo ocorrer também pela redução da quantidade de Oxalobacter formingens, bactéria intestinal responsável pela degradação do oxalato. Ademais, os ácidos biliares e os próprios ácidos graxos aumentam a permeabilidade do cólon ao oxalato. Associado a essas alterações é comum que ocorra uma redução do volume urinário em consequência da diarreia ${ }^{5}$.

A Oxalobacter formingens é uma bactéria anaeróbica gram - negativa que participa da metabolização de oxalato no trato intestinal, através da degradação do oxalato pela enzima oxalyl-CoA descarboxilase ${ }^{14}$, e sua colonização está associada com uma redução em $70 \%$ do risco de se desenvolver litíase renal por oxalato de cálcio, já que o oxalato urinário está diretamente proporcional á nefrolitíase ${ }^{15}$.

Indivíduos com excreção urinária maior de oxalato apresentam níveis mais baixos de Oxalobacter formingens, sendo a ausência da bactéria degradante do oxalato na flora intestinal um fator de risco para hiperoxalúria e, consequentemente, aumento na probabilidade de urolitíase ${ }^{14 ; 16}$. 
Além da deficiência de fatores que contribuem para prevenção da nefrolitíase, pacientes com DC apresentam uma perda líquida significativa, sendo este fator promotor para tal complicação, confirmando, portanto, a perda líquida em excesso que ocorre nessas situações é verificada em estudos a ocorrência de 6 á 9 evacuações por dia nesses pacientes ${ }^{17}$.

Com a diarreia constante e, consequentemente, a perda líquida, ocorre ainda alterações em vários outros nutrientes importantes na gênese da litíase.

Os pacientes perdem grande quantidade de líquido intestinal alcalino e os rins respondem secretando urina ácida e concentrada. Esses fatos colaboram para o aparecimento de cálculos de ácido úrico. A hiperuricemia nesses doentes predispõe à formação de cálculos de cálcio ${ }^{18}$ devido á maior reabsorção óssea e menor reabsorção tubular renal de cálcio por consequência da queda no $\mathrm{pH}$ endógeno, como forma de controlar a acidez.

As condições como doença inflamatória intestinal resultam em urina ácida por desidratação e perda gastrointestinal de bicarbonato por diarreia, predispondo a formação de cálculos de ácido úrico ${ }^{19}$.

Os fatores mais frequentemente implicados na litogênese são: desidratação, oligúria, menor absorção de água, obstrução e/ou infecção do trato urinário, excreção anormal de ácido úrico, urina ácida, alterações na absorção e excreção de oxalatos, uso de corticoides, hipercalciúria, absorção intestinal diminuída de sódio com concomitante decréscimo no sódio urinário ${ }^{18}$.

De acordo com o estudo citado, em pacientes submetidos à ressecção de algum segmento ileal à excreção de fatores inibidores (citrato e magnésio) foi menor e dos fatores promotores (cálcio, oxalato, ácido úrico, fósforo e sódio) foi maior. Considerando que tanto o citrato quanto o magnésio são importantes agentes inibidores da cristalização, suas deficiências podem favorecer o processo de crescimento e agregação dos cristais, principalmente dos sais de cálcio 5 .

Entre as diversas causas de hipocitratúria provavelmente a mais importante no presente contexto é a hipomagnesúria. A explicação seria que nos túbulos renais ocorre a formação de citrato de magnésio reduzindo assim a reabsorção urinária de citrato e aumentando a excreção urinária de citrato. Na deficiência de magnésio, uma maior concentração de citrato está disponível para reabsorção, resultando assim em menor excreção urinária de citrato ${ }^{20}$.

Ocorre hipocitratúria para valores de citrato urinário inferiores a $320 \mathrm{mg} / \mathrm{dia}^{21}$. O citrato tem dois efeitos importantes na prevenção da nefrolitíase conhecidos como efeitos solubilizante e inibidor da cristalização dos sais de cálcio ${ }^{22}$, dessa forma, a hipocitratúria isolada ou combinada com outras alterações metabólicas é um agente da nefrolitíase.

O mecanismo pelo qual o citrato é um fator inibidor seria que o mesmo forma um cálcio complexo na urina, dessa forma, menos cálcio está disponível para ligar o oxalato urinário, o que ajuda a prevenir a formação de cálculos de oxalato de cálcio ou de fosfato de cálcio ${ }^{19}$.

Com relação às proteínas e ao sódio, eles são implicados como importantes fatores de risco para a formação de cálculos. $\mathrm{O}$ sódio pode provocar hipercalciúrias por intercâmbio tubular renal com o cálcio, e as proteínas determinam uma sobrecarga ácida, afetando a composição da urina, ao aumentar a excreção de cálcio e oxalato e diminuir a excreção de citrato e o $\mathrm{pH}$ urinário. A acidez urinária pode ser um fator determinante, pois para um $\mathrm{pH}$ $<5,5$ a solubilidade do ácido úrico perde-se e o processo de cristalização é potenciado ${ }^{21}$.

Contudo, percebem-se várias alterações metabólicas que envolvem o curso da DC, o que demonstra a importância de se conhecer todos esses fatores, em que o diagnóstico nutricional e posterior tratamento são de extrema importância para manutenção da qualidade de vida desses pacientes.

\section{Tratamento nutricional}

A DC, pelo seu envolvimento do trato gastrintestinal e seus efeitos sobre a ingestão alimentar, é associado á deficiência nutricional, podendo provocar até desnutrição severa, com grande perda de peso, além de desencadear complicações como a nefrolitíase, devido á grande perda líquida e deficiência na absorção de nutrientes.

Os objetivos do tratamento nutricional são a correção e a prevenção de déficits nutricionais, assim como o controle e alívio dos 
sintomas.Portanto, o diagnóstico nutricional dietético é de extrema importância, pois, confirma a relação entre a adequação da ingestão de calorias e nutrientes x déficit nutricional/ metabólico e/ou a queda no estado geral apresentado pelo doente ${ }^{23}$.

É crucial a determinação do grau de severidade ou atividade da doença. A severidade, a duração e a localização da doença são importantes na resposta ao tratamento.

É importante ressaltar que a tolerância individual deve ser considerada, sempre com o objetivo de preservar um bom estado nutricional, pois, este pode ser fator de agravamento da doença, ou influir positivamente na recuperação ${ }^{24}$.

Para que isso ocorra, é fundamental a escolha da via de administração da dieta adequada para cada fase da doença de acordo com paciente, neste estudo será abordado somente a via oral.

A melhor maneira de melhorar a ingestão oral é evitar grandes restrições dietéticas e estimular a utilização de alimentos que sejam preferência do paciente, evitando retirar da dieta alimentos cujo efeito nocivo não têm sido demonstrado ser capaz de agravar a doença, a fim de atingir, com maior facilidade, as necessidades calórico-proteicas ${ }^{25}$.

Uma ingestão calórica diária de 35 a 40 $\mathrm{Kcal} /$ dia de peso ideal e 1 á $1,5 \mathrm{~g} / \mathrm{kg}$ do peso ideal de proteína/dia satisfaz as demandas de proteína e energia da maioria dos pacientes adultos com a doença ativa e existem ainda novas possibilidades de intervenção nutricional com a utilização dos ácidos graxos ômega 3 e probióticos ${ }^{26}$.

A terapia nutricional deve ser da seguinte maneira: hipolipídica $(<20 \%$ das calorias totais); na fase aguda, evitar alimentos fonte de lactose e controlar mono e dissacarídeos, dieta rica em fibras solúveis e pobre em insolúveis; na fase de remissão, evoluir o teor de fibras insolúveis; evitar alimentos flatulentos, oferecer nutrientes específicos (glutamina, ácido graxo ômega 3 e arginina e glutamina $)^{27}$.

\subsection{Glutamina}

A glutamina participa de processos metabólicos importantes tais como: síntese de proteínas, gliconeogênese, homeostase ácido- base e biossíntese de ácidos nucleicos, sendo utilizada também como substrato pelas células da mucosa intestinal (enterócitos) e células imunológicas ${ }^{28}$.

Em contrapartida, há relatos da piora da atividade da DC e aumento da permeabilidade intestinal em pacientes com uso de glutamina. Parece que o mecanismo responsável por esta piora é a síntese de arginina á partir da glutamina, com consequente liberação de óxido nítrico, que provocaria o aumento da permeabilidade vascular intestinal ${ }^{29}$.

Nesse ponto, entende-se, portanto, a necessidade de mais estudos científicos para real contribuição e recomendações de ingestão diária da glutamina em pacientes com DC.

\subsection{Probióticos}

Os probióticos podem, portanto, inibir a proliferação de organismos patogênicos, diminuir a incidência, duração e gravidade de doenças gástricas e intestinais. $\mathrm{Na}$ função imunológica, estimulam a resposta específica e inespecífica, mediada pelo aumento da concentração de imunoglobulinas ${ }^{30}$; amenizam a intolerância á lactose; controlam a diarreia aguda; melhoram a atividade clínica da doença e previnem as recidivas ${ }^{26}$.

Para que ocorra a manutenção da saúde do trato gastrintestinal, é recomendado a ingestão de 1 a 2 bilhões de unidades formadoras de colônia/ dia, existentes em leites fermentados ${ }^{31}$.

\subsection{Fibras solúveis e Citrato}

Com relação às fibras solúveis, sua ingestão diária recomendada é de 5 a $10 \mathrm{~g} / \mathrm{dia}$, sendo representadas pela pectina (frutas) e pelas gomas (aveia, cevada e leguminosas: feijão, grão de bico, lentilha e ervilha) ${ }^{32 . ~ . ~ E s s e ~}$ nutriente sofre fermentação no interior intestinal produzindo os ácidos graxos de cadeia curta (acetato, propionato e butirato) nutrientes imunomuduladores que atuam como fonte de energia para os colonócitos, estimulando o trofismo intestinal e aumentando a reabsorção de água e eletrólitos pelas células intestinais ${ }^{33} \mathrm{e}$ complementando a deficiência de fibras dietéticas, exercem papel na gênese da nefrolitíase por metabolismo do citrato, uma vez que exis-

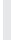


te relação positiva entre a baixa excreção de citrato e baixa ingestão de fibras ${ }^{34}$.

Ao aumentar os níveis de citrato urinário, determina-se uma redução da saturação de oxalato e fosfato de cálcio ${ }^{21}$.

\subsection{Carboidratos}

O consumo de carboidratos pode elevar a excreção urinária de cálcio e oxalato. Acredita-se que o excesso de carboidratos na alimentação diminuiria a absorção de fosfato intestinal, levando a hipofosfatemia e, consequentemente, aumento do calcitriol plasmático implicando em aumento da absorção intestinal de cálcio e simultâneo aumento de absorção de oxalato. Parece também que os carboidratos estimulariam a síntese endógena de oxalato, que será posteriormente, excretado na urina. Além do mais, muitos alimentos ricos em carboidrato são ricos em oxalato ${ }^{34}$.

Além disso, devido a DC existem lesões nas vilosidades da mucosa intestinal que leva a uma diminuição de diversas enzimas, modificando a abrsorção intestinal de carboidratos, sendo a lactase a última a se regenerar ${ }^{35}$, o que torna prejudicada a absorção de derivados do leite .

Portanto, é necessário que se controle a ingestão de carboidratos nesses pacientes a fim de evitar maiores desconfortos gastrintestinais e urogenitais.

Sabe-se que o intervalo de concordância usualmente aceito á respeito da recomendação da ingestão diária de carboidrato é de 50 á $60 \%$ do valor energético total (VET) e de 4 a 6 gramas/Kg de peso ideal /dia sendo utilizado para indivíduos saudáveis.

$\mathrm{Na}$ DC existe uma deficiência de estudos que informe tal recomendação, o que torna necessárias mais investigações atendendo a DC e Nefrolitíase.

\section{5. Ácido graxo ômega 3}

Em se tratando de ácido graxo ômega 3 (AGn - 3), em estudo realizado com ratos, concluiu-se que os animais com DII que receberam suplementação de AGn - 3 apresentaram menos alterações inflamatórias, maior preservação da integridade da mucosa e ausência de úlceras ${ }^{36}$.
A suplementação de óleo de peixe em 2,7g de ácido graxo n-3 distribuídos em 9 cápsulas, pode ser necessária, pois, reduzem a frequência dos surtos da doença e aumentando os intervalos ${ }^{37}$.

Os Ácidos graxos poli-insaturados (AGPI) do tipo ômega-3 produzem mediadores lipídicos chamados eicosanoides com potencial de diminuir/inibir o efeito das citocinas pró-inflamatórias. Dessa maneira, tem sido proposto que a suplementação ou ingestão adequada desse tipo de AGPI pode beneficiar pessoas com doenças inflamatórias crônicas, portanto, sua utilização pode ser efetiva na manutenção da remissão da doença de $\mathrm{Crohn}^{38}$.

Os benefícios são muitos, porém, são necessários mais estudos para investigar o papel desse nutriente como suplemento dietético.

\subsection{Vitamina C, Oxalato e Cálcio}

O oxalato é o produto final do metabolismo da glicina, $(40 \%$ a $50 \%$ da síntese hepática) e $40 \%$ resultante do metabolismo do ácido ascórbico, sendo que o oxalato dietético contribui com uma fração de $20 \%$ do oxalato urinário39.

Por isso, indivíduos formadores de cálculo são frequentemente aconselhados a evitar suplementos de vitamina $C$, não ultrapassando a recomendação de $60 \mathrm{mg} /$ dia. No entanto, estudos sobre a excreção urinária de oxalato, após carga de vitamina $\mathrm{C}$, têm produzido resultados inconsistentes, pois, o aumento de oxalato urinário observado após experimental administração de altas doses de vitamina $\mathrm{C}$ parece ser devido à conversão in vitro de ascorbato para oxalato durante o procedimento analítico e não in vivo ${ }^{40}$.

Apesar de estudos identificarem maior excreção de oxalato em indivíduos que ingerem suplemento de vitamina $C$, são necessários mais estudos para identificar a real atuação desse nutriente na nefrolitíase.

Dessa forma, além da contribuição do oxalato dietético, o cálcio dietético influencia na biodisponibilidade do oxalato ingerido, sendo importante fator na formação de cálculos de oxalato de cálcio.

O oxalato é absorvível somente na sua forma solúvel, e sua absorção é possível no estômago, intestino delgado e cólon ${ }^{41}$. 
Percebe-se que embora a DC possa acometer qualquer parte do trato gastrintestinal, esta se instala com maior frequência no intestino delgado e a absorção de oxalato ocorre também nesta área, o que favorece a alteração absortiva citada anteriormente.

Os alimentos de alto teor de oxalato não devem ser evitados com base apenas no seu teor de oxalato já que uma quantidade limitada é biodisponível, entretanto, os alimentos com baixo teor de oxalato e uma maior biodisponibilidade podem aumentar notavelmente o oxalato urinário ${ }^{19}$.

O oxalato está presente em grande quantidade nos alimentos de origem vegetal, somente espinafre e ruibarbo são considerados alimentos de alto risco, pois apresentam grande concentração de oxalato biodisponível. Amendoim, amêndoa, chocolate, noz pecã e chá instantâneo são considerados alimentos de moderado risco $^{42}$.

Complementando, muitos alimentos à base de soja apresentam alta concentração de oxalato (mais de $10 \mathrm{mg} /$ porção), o que seria contraindicado para pacientes formadores de cálculos. Contudo, por apresentarem alta concentração de fitato, um provável protetor na gênese da litíase, os alimentos à base de soja que contêm menores concentrações de oxalato podem ser benéficos nesses pacientes ${ }^{43}$.

Uma boa estratégia nutricional seria então oferecer $1200 \mathrm{mg}$ de cálcio/dia como preconiza a Organização Mundial da Saúde (OMS) e diminuir a oferta de alimentos fonte de oxalato, prevenindo assim a hiperoxalúria. Outra opção seria a submissão dos alimentos fontes de oxalato á cocção a fim de reduzir seus níveis ${ }^{44}$.

É importante destacar que em fases ativas da DC, a absorção de lactose fica prejudicada, tornando necessário a substituição por outros alimentos toleráveis fonte de cálcio, como a sardinha.

\subsection{Líquidos}

Outro fator importante é a oferta de líquidos, já que na medida em que se aumenta a ingestão de líquido diminui a supersaturação urinária de oxalato ${ }^{45}$.
O risco de formação de cálculo aumenta quando o volume urinário é menor que $1 \mathrm{~L} /$ dia e reduz quando é maior que $2,5 \mathrm{~L} / \mathrm{dia}^{46}$.

Deve-se orientar o paciente a ingerir aproximadamente $30 \mathrm{ml} / \mathrm{kg} /$ dia de líquidos, de preferência água, sucos de fruta como os de limão e laranja, por conter elevado teor de citrato, e chás de frutas, flores ou ervas a fim de elevar o volume urinário e diminuir o risco de formação de cálculos ${ }^{47}$.

Com relação á ingestão de água mineral- esta, apesar de aumentar a hipercalciúria, o magnésio e o bicarbonato, devido aos seus componentes -leva a mudanças favoráveis no pH urinário, com aumento da excreção de magnésio e citrato, inibidores da formação de cálculo por oxalato de cálcio ${ }^{48}$.

Além da prevenção da litíase renal, a ingestão de líquido é importante também para manter ou recuperar a quantidade perdida nas evacuações frequentes.

\subsection{Magnésio}

A ingestão de cálcio e magnésio pode moldar a absorção intestinal de oxalato ${ }^{49}$, sendo que a ingestão de magnésio pode diminuir a excreção de oxalato de maneira semelhante ao cálcio ${ }^{50}$, ou seja, se ligando ao oxalato e aumentando assim a excreção de citrato e pH urinário ${ }^{51}$.

As quantidades recomendadas de magnésio em pessoas adultas saudáveis são de $400 \mathrm{mg} /$ dia para sexo masculino e $310 \mathrm{mg} / \mathrm{dia}$ para sexo feminino, sendo que não existem recomendações especiais para doenças específicas, porém, em pacientes que apresentam uma ou mais das condições que aumentam as perdas de magnésio, recomenda-se um acompanhamento sistemático dos níveis sanguíneos, suplementando sempre que necessário ${ }^{52}$.

\subsection{Proteína}

A ingestão protéica está diretamente relacionada com aumento dos fatores de risco litogênicos. Os principais fatores envolvidos seriam a ingestão de proteínas como causa de hiperuricosúria,devido à sobrecarga de purinas; hiperoxalúria pelo aumento da síntese de oxalato;e hipocitratúria em função da maior reabsorção tubular de citrato. Pode levar ainda

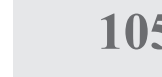

\section{.}

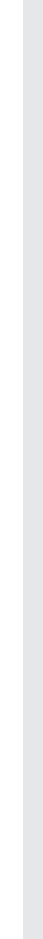

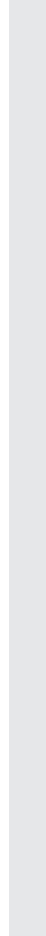

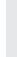

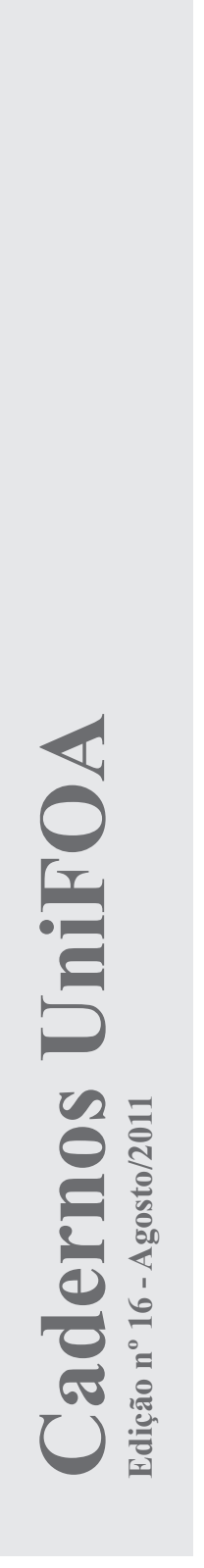


à hipercalciúria por promover aumento da reabsorção óssea e diminuir a reabsorção tubular renal de cálcio, devido a uma maior excreção de ácidos diminuindo o $\mathrm{pH}$ urinário ${ }^{42 ; 51 ; 53}$.

Uma explicação para redução de citrato seria que este é um componente chave no Ciclo de $\mathrm{Krebs}^{46}$.

Existem três teorias para a formação de cálculo renal: a primeira seria que o ácido úrico absorve os inibidores do oxalato de cálcio; a segunda, é que na urina supersaturada, a semelhança na estrutura do cristal promove o crescimento epitaxial do oxalato de cálcio no ácido úrico e a terceira seria que o ácido glutâmico adere á sobreface do cristal de ácido úrico, ajudando íons de cálcio e oxalato a se agregarem e cristalizarem.

Portanto, sua ingestão deve ser controlada a quantidades citadas anteriormente, em casos em que o paciente não apresenta desnutrição.

\subsection{Sódio}

O sódio eleva a excreção de cálcio já que ambos são reabsorvidos no túbulo proximal e alça de Henle, provocando, consequentemente, hipercalciúria, sendo este um fator de risco para litogênese, além de reduzir a excreção urinária de citrato. Esse efeito é aditivo quando associado ao aumento no consumo de proteínas. Sugere-se a redução do sódio na dieta para 4 a 5 g/dia, para que não ocorra excreção superior a $100 \mathrm{mEq}^{51}$.

Para cada $100 \mathrm{mEq}$ de aumento no consumo de sódio pela dieta, há um aumento da excreção urinária de cálcio em $25 \mathrm{mg}^{42}$.

\subsection{Potássio}

Com relação à ingestão de potássio, sua ingestão é inversamente proporcional ao risco de cálculos renais, já que, aumenta a reabsorção tubular de cálcio e aumenta a excreção de citrato, portanto, formadores de cálculo devem aumentar esse nutriente em suas dietas, escolhendo frutas e vegetais de baixo teor de oxalato ${ }^{19}$.

\subsection{Suplementos alimentares}

Os suplementos nutricionais especializados auxiliam o manejo nutricional de pacientes em situações clínicas que seriam dificilmente contornadas com os alimentos e suplementos convencionais ${ }^{23}$.

As vitaminas e minerais suplementares podem ser necessários devido ao fato de pacientes cronicamente doentes ter necessidade de evitar alguns tipos de alimentos ou certos grupos alimentares ou para corrigir as interações de drogas e nutrientes.Pequenas quantidades de suplementos isotônicos, líquidos, orais podem restaurar a ingestão, e, em casos de má absorção de gorduras, a suplementação ou alimentos fonte de triglicerídeos de cadeia média (TCM) podem ser eficazes no fornecimento de calorias e como veículos para nutrientes lipossolúveis ${ }^{4}$.

Dentre a deficiência de vitaminas lipossolúveis, a mais relatada é a vitamina $\mathrm{D}$, graças a deficiência absortiva de gordura, podendo predispor uma suplementação.

Pode ocorrer também deficiência de vitamina B12 devido à má absorção tornando necessária a suplementação deste mineral.

Além da vitamina B12, é indicado também a oferta de B6, já que o uso fisiológico ou farmacológico na dose de 10 a 500mg/dia pode está relacionada com a diminuição da hiperoxalúria $^{40}$.

A suplementação de citrato como terapia farmacológica é muito interessante, pois, este age como um quelante que se liga ao cálcio ionizado disponível na urina, inibindo o crescimento e a agregação de cristais urinários com oxalato de cálcio, além de promover a alcalinização urinária e consequentemente repor o citrato em pacientes com hipocitratúria ${ }^{54}$. Porém epigastralgia, pirose, diarreia e plenitude gástrica são queixas frequentes e limitam a terapêutica ${ }^{13}$.

\section{Conclusão}

Conclui - se neste artigo que a DC possui correlação com a nefrolitíase, devido às alterações absortivas encontradas, sendo necessário que as deficiências nutricionais sejam detectadas o mais cedo possível, para que se possa traçar um plano alimentar individualizado de acordo com o estado nutricional do paciente. Assim, os efeitos deletérios da doença poderão ser atenuados, proporcionando a recuperação e manutenção do estado nutricional do paciente e melhorando a sua qualidade de vida. 
É importante salientar que há necessidades de maiores estudos, pois não existem ainda diretrizes nutricionais para nefrolitíase em pacientes com DC, contudo, parece ser possível conseguir uma resposta satisfatória desses pacientes com relação ao tratamento nutricional, retardando assim, o aparecimento da nefrolitíase e proporcionando uma vida normal durante um maior período, pois, a dieta adequada ás necessidades do indivíduo traz importantes benefícios para a evolução e tratamento da DC e nefrolitíase, apresentando, portanto, um efeito direto na progressão das doenças.

É importante ressaltar ainda a importância da atuação do nutricionista na elaboração de dietas que atendam ás necessidades nutricionais, devido às características restritivas na alimentação impostas pelas duas doenças isoladamente e em conjunto. Dessa forma, as dietas devem ser individualizadas para que se possa adquirir a adesão ao tratamento de forma adequada.

\section{Bibliografia}

1. SOUZA,Marcellus Henrique et al. Evolução da ocorrência (1980-1999) da doença de Crohn e da Retocolite Ulcerativa Idiopática e análise das suas características clínicas em um hospital universitário do sudeste do Brasil.Arq. Gastroenterol. São Paulo. 2002. jun 2.(39).

2. LOFTUS,Conor et al. Update on the incidence and prevalence of Crohn's disease and ulcerative colitis in Olmsted County, Minnesota, 1940-2000.J. Inflam. Bow. Dise.2007.13(3):254-61,

3. SOUZA, Mardem;BELASCO,Angé lica;NASCIMENTO,José Eduardo. Perfil Epidemiológico dos Pacientes Portadores de Doença Inflamatória Intestinal do Estado de Mato Grosso. Rev bras Coloproct. 2008 ago/set; 3.(28):324-328.

4. PETER, L. Terapia Clínica Nutricional paraDistúrbiosdoTratoGastrointestinal Baixo. In:MAHAN, L KATHLEEN et al.Alimentos, nutrição e dietoterapia. São Paulo: Roca, 2005:657-662.
5. VIANA.Lopes.M.L.V, et al. Doença de Crohn e cálculo renal: muito mais que coincidência?. Arq.Gastroenterol, 2007 jul./set; 3(44).

6. TOSTES,V;CARDOSO,L.R.Recentes avanços em litíase urinária. J Bras Nefrol . $2001 \mathrm{mai} / \mathrm{jun} ; 23(3): 166-73$.

7. ALVES, Rui et al;Ocorrência de litíase renal em pacientes com ressecção parcial do intestino delgado.J.Bas Nefrol. 2002 jan/fev; 22. (2):55-62,.

8. GRIF F I TH S, A N N E. D o e n ç a Inflamatória Intestinal in, SHILLS, Maruce. et al.Tratado de Nutrição Moderna na Saúde e na Doença.Manole, 2003. p. 1222 .

9. CÉSAR, Júlio; S ANT OS, Júnior. Doença de Crohn - Aspectos Clínicos e Diagnósticos. Rev. Brás. Coloprocto. 1999 out/dez;4(29):276-285.

10. ELIA, Paula et al. Análise descritiva dos perfis social, clínico, laboratorial e antropométrico de pacientes com doenças inflamatórias intestinais, internados no Hospital Universitário Clementino Fraga Filho, Rio de Janeiro.Arq. Gastroenterol. 2007 Oct./Dec;4(44).

11. SUMANDA,Kane. Urogenital Complications of Crohn's Disease. The Ame. J. Gastroentero. 2006 Dec;3(101):640-643.

12. PERES,Luis;CARVALHO,Carlos Alberto.Relato de Caso: Ocorrência de doença de Crohn em uma paciente com cistinúria e retardo mental. Bras Nefrol . $2001 \mathrm{mar} / \mathrm{abr} ; 23(4): 221-23$.

13. CARVALHO, MURÍCIO. Nefrolitíase in: RIELLA, Miguel Carlos; MARTINS, Cristina:Nutrição e o Rim. Rio de Janeiro: Guanabara Koogan, 2001. p.615 - 617.

14. SCOTT, Toxel etal.Intestinal Oxalobacter formigenes Colonization in Cálcium Oxalate Stone Formes and its Ralation to Urinary Oxalate.J. Endouro.. 2003 april 3(17):173-176.

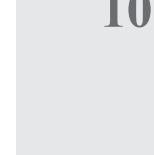

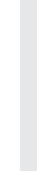

\section{.}


15. KAUFMAN,David.W et al. Oxalobacter formigenes May Reduce the Risk of Calcium OxalateKidney Stones.J.Ame.Soci.Nephr. 2008. Dispnível em: http://jasn.asnjournals. org/cgi/rapidpdf/ASN.2007101058v1.pdf Acesso em: 13/03/2009

16. KAZUO,M et al. Association of Absence of Intestinal Oxalate Degrading Bacteria With Urinary Calcium Oxalate Stone Formation. Intern. J. Urol. 2003 jun 6(10):293-296.

17. SALVIANO,Flávia.N;BURGOS,Maria Goretti;SANTOS, Eduila. Perfil socioeconômico e nutricional de pacientes com doença inflamatória intestinal internados em um hospital universitário. Arq. Gastroenterol. 2007 abr./Jun;2(44).

18. TEIXEIRA, Willian et al, Manifestações Extra-Intestinais após Tratamento Cirúrgico da Retocolite Ulcerativa. Rev bras Coloproct. 2001 mar/abr;1(21):9-18.

19. KATY,Wilkens;:Terapia Nutricional para Distúrbios Renais. In:MAHAN, L KATHLEEN et al.Alimentos, nutrição e dietoterapia. São Paulo: Roca, 2005.p.657-662.

20. MCCONNELL, N et al.Risk factors for developing renal stones in inflammatory bowel disease.BJU International. 2002 jan;89:835-841.

21. GOMES, Pedro.Profilaxia da Litíase Renal.Acta Urológica. 2005 mai/jun; 22(3):47-56.

22. SAKUNO,Marle Leiko et al.Citrato urinário na nefrolitíase. Rev Bras. Anál. Clin.. 2002;34(3):169-171.

23. BAXTER,Yara;WAITZBERG, Dan. Indicações e Usos de Suplementos Nutricionais Orais.in, WAITZBERG, Dan.Nutrição Oral, Enteral e Parenteral na Prática Clínica:Atheneu, 2006.p.546.

24. NEVES, Fabrícia; KIFMAN, Rosalina; MATTOS, Inês. Mortalidade por câncer de cólon e reto e consumo alimentar em capitais brasileiras selecionadas.Arqui. Gastroenterol.. 2005 Jan/mar;1(42).
25. JÚNIOR, Paulo. Molestia inflamatória intestinal.P1361-1363inWAITZBERG,Dan Nutrição oral, enteral e parenteral na prática clínica..São paulo, Atheneu 2006.1858p

26. FLORA,Ana.L;DICHI,Isaias.Aspectos atuais na terapia nutricional da doença inflamatória intestinal.Rev Brás. Nutr. Clín., 2006 fev.2(21):131-137.

27. CARUSO,Lúcia.Distúrbios do Trato Digestório in, Cupari, Lílian. Guia de Medicina Ambulatorial e Hospitalar UNIFESP/ Escola Paulista de Medicina. Barueri:Manole,2002.p.208-210.

28. BARRUAJ,Maria.C et al.Terapia Nutricional na Doença Inflamatória Intestinal.The Electronic Journal of Pediatric Gastroenterology, Nutrition and Liver Diseases.2004. 11 p.Disponível em: < http://www.e-gastroped.com.br/dec04/ indexdec04.htm> Acesso em: 9 .mar.2004.

29. AKONBENG,A.Doubleblindrandomized controlled trial of glutamine-enriched polymeric diet in the treatment of Crohn's disease. J. Pedi. Gastroenterol., Nutria. Liv. Disea.. 2000 jul/ago;1(30):78 - 84.

30. COLLI,C et al.Alimentos Funcionais in, CUPARI, Lílian. Guia de Medicina Ambulatorial e Hospitalar UNIFESP/ Escola Paulista de Medicina. Barueri:Manole,2002.p.55-63.

31. American Dietetic Association. Position of the American Dietetic Association: Functional Foods. J. Ame. Diet. Assoc; 2004; 5(104).

32. SPOSITO,Andrei et al.IV Diretriz Brasileira Sobre Dislipidemias e Prevenção da Aterosclerose Departamento de Aterosclerose da Sociedade Brasileira de Cardiologia Coordenação Geral. Arqu. Brás. Cardiol.. 2007 abr;1(88)

33. LACERDA,Fábio;PACHECO,Marcos Tadeu. X Encontro Latino Americano de iniciação Científica e VI Encontro Latino Americano de Pós - Graduação - Universidade do Vale do Paraíba. Itajubá. MG,2004. 
34. SILVA, José Augusto.;CORREIA,Maria Isabel.Nutrição e Litíase.Revista Virtual de Medicina.1999. Dispnível em: $<$ http://www.medonline.com.br/nutlit. htm>Acesso em: 14/06/2008.

35. FARIAS,Fábio;NETO, Ulisses. Intolerância aos Carboidratos. The Electronic Journal of Pediatric Gastroenterology, Nutrition and Liver Diseases.2003.10 p. Disponível em: $<$ http://www.e-gastroped.com. br/dec04/intolerancia.htm $>\quad$ Acesso em:01.05.2009.

36. CAMPOS,Fábio

Guilherme. Imunonutrição em Colite Experimental: efeitos Benéficos dos Ácidos Graxos ômega - 3.Arqu. Gastoenterol, 2002 jan/mar; 1(39):48 - 54.

37. SIMÕES,MARIA et al. Opções TerapêuticasParaasDoenças Inflamatórias Intestinais: Revisão.Rev. Brás. Colop. $2003 \mathrm{jul} / \mathrm{set} ; 3(23): 172-182$.

38. CAMPOS, Letícia. Tipo de Dieta Pode Influenciar Pacientes com Doenças Inflamatórias Intestinais?.2008. Disponível em: http://www.nutritotal. com.br/newsletter/index.php. Acesso em: 20 mar,2009.

39. HOLMES,Ross;GOODMAN,Harold;A SSIMOS,Dean.Contribution of dietary oxalate to urinary oxalate excretion. Kid. Interna, 2001 jul/aug;59:270-276.

40. CURHAN,GARY et al. Intake of Vitamins B6 and $\mathrm{C}$ and the Risk of Kidney Stones in Women. J. Ame. Soci. Nephrol, 1999 oct.10:840-845.

41. JAEGER,P.H;ROBERTSON,W.G.Role ofDietary Intake and Intestinal Absorption of Oxalate in Calcium Stone Formation.J. Nephrol. Phys.. 2004;98:64-71.

42. MELLO, Elza; SCHNEIDER,Márcia . A Importância da Dieta No Manejo da Hipercalciúria. Rev. do Hosp. Clín. P.A. 2006 ago;2(26):52-60.
43. AL-WAHSH,Ismail et al.Oxalate and Phytate of Soy Foods.J. Agric. Food Chemi.. 2005 jun; 14(53):5670 - 5674.

44. GERALDO,Ana,Paula.G. Alimentação e Litíase Renal,2006.Disponível em: $<$ http://www.nutrociencia.com.br/ upload files/arquivos/Cuidados $\% 20$ com> Acesso em:27/02/2009

45. SERRA,Adelaide et al.Avaliação Metabólica da Lítiase Cálcica Idiopática Recorrente em Portugal.ACTA Médica Portuguesa 2002 nov;17:27-34.

46. OLIVEIR A, E 1 i a n e; F R EITAS, $\mathrm{C} 1 \mathrm{ara}$; T E O D Ò S I O, M a r t a 1 . Nutrientes,Líquidos e Fibras na Formação de Cálculos Renais.Rev Brás. Nut. Clín. 2003set/out;3(18):142 - 148.

47. CUPPARI, et al.Doenças Renais in, CUPARI, Lílian. Guia de Medicina Ambulatorial e Hospitalar UNIFESP/ Escola Paulista de Medicina. Barueri:Manole, 2002.p.197.

48. SIENER, R;JAHNEN,A;HESSE,A. Influence of a Mineral Water Rich in Calcium,Magnesium and Bicarbonate on Urine Composition and The Risk of Calcium Oxalate Crystallization.Euro. J. Clin. Nut. 2004apr;3(58):270 - 276.

49. HOLMES,Ross;ASSIMOS,Dean.The Impact of Dietary Oxalate on kidney Stone Formation.J. Urol. Resea. 2004; 32:311-316.

50. TAYLOR,Eric \& CURHAN,Gary. Oxalate Intake and the Risk for Nephrolithiasis.J Am Soc Nephrol.2007 apr;18:2198-2204.

51. LEMOS,G.C;SHOR,N.Litíase Urinária: Aspectos Metabólicos Em adultos e Crianças.Associação Médica Brasileira e Conselho Federal de Medicina. jun. 2006. Disponível em: <www.projetodiretrizes. org.br/5_volume/29-LitiUrin.pdf > Acesso em: 19/03/2009. 
52. CASTILHO,Anna Cristina et al.Cálcio e Magnésio,2005.Acesso em 23/03/2009. Disponível em: $<$ http://www.amway.com. br/downloads/misc/Calcio_e_Magnesio_ IMEN.pdf> Acesso em 23 mar,2009.

53. BATISTA,Rodrigo et al. Nutrição e Saúde Perspectivas Revisitadas.J. Brás. Méd.2008 mar/abr; 5:12-25.

54. ARCANGELA,Amélia et al.Estudo da excreção urinária de cálcio, potássio e sódio com o emprego de citrato de potássio na hipercalciúria idiopática na criança.Rev Paul. Pedia. 2007 mai/ jun;2(25):119-123.

\section{Endereço para Correspondência:}

Alden Santos Neves

aldens@uol.com.br

Centro Universitário de Volta Redonda - UniFOA

Avenida Paulo Erlei Alves Abrantes, 1325

Três Poços - Volta Redonda - RJ

CEP: $27240-560$

Informações bibliográficas:

Conforme a NBR 6023:2002 da Associação Brasileira de Normas Técnicas (ABNT), este texto científico publicado em periódico eletrônico deve ser citado da seguinte forma: ALMEIDA, Nívea Veiga; NEVES, Alden Santos. Doença de Crohn e sua relação com a Nefrolitíase: Tratamento Nutricional. Cadernos UniFOA. Volta Redonda, Ano VI, n. 16, agosto 2011. Disponivel em: <http://www.unifoa.edu.br/cadernos/edicao/16/99.pdf> 